\title{
Study design of the International Stroke Trial (IST), baseline data, and outcome in 984 randomised patients in the pilot study
}

\author{
International Stroke Trial Pilot Study Collaborative Group
}

\begin{abstract}
Background and purpose-To test the design and feasibility of a very large randomised controlled trial assessing the efficacy and safety of antithrombotic therapy started within 48 hours of symptom onset in patients with suspected acute ischaemic stroke.

Design-Randomised controlled multicentre open study, with a $3 \times 2$ factorial design, allocating patients to: medium dose subcutaneous heparin (12 500 units twice per day), versus low dose subcutaneous heparin (5000 units twice per day) versus no heparin; and aspirin $(300 \mathrm{mg}$ daily) versus no aspirin. Treatment was given for two weeks or until discharge from hospital if sooner.

Results-984 patients were randomised. CT was performed in 924 (94\%) (before randomisation in $622 / 984(63 \%))$. Within 14 days: 97 patients had died (10\%), 30 (3.0\%) had a fatal or non-fatal recurrent ischaemic stroke, nine $(0.9 \%)$ had fatal or non-fatal recurrent stroke due to intracranial haemorrhage, and eight $(0 \cdot 8 \%)$ had a fatal or non-fatal pulmonary embolus. At six months, vital status was known for 975 patients (99\%), of whom $210(22 \%)$ were dead, $373(38 \%)$ were alive but dependent, and 225 (23\%) were independent but not fully recovered.
\end{abstract}

Conclusions-The trial procedures proved practicable and a wide variety of patients were recruited. Sample size calculation based on the event rates confirmed that reliable evidence on the balance of risk and benefit of early antithrombotic therapy might require a study with more than 20000 patients. Recruitment rates in the pilot study indicated that if about 200 hospitals participated, recruitment could be completed by 1997.

\section{(F Neurol Neurosurg Psychiatry 1996;60:371-376)}

Keywords: aspirin; heparin; acute ischaemic stroke; randomised clinical trial

Correspondence to:

Dr P A G Sandercock, Department of Clinical Neurosciences, Western General Hospital, Edinburgh EH4 2XU, UK

Received July 1995 and in final revised form 29 November 1995 Accepted 1 December 1995

No specific medical treatment has yet been shown to be effective for routine use within the first 48 hours of the onset of symptoms of stroke. ${ }^{1}$ Whereas some of the completed randomised controlled trials have been large enough to exclude a large benefit or a large hazard from treatment, none was large enough to confirm or refute a moderate benefit (or moderate hazard). ${ }^{2}$ As stroke is common and is often fatal or disabling, ${ }^{3}$ a treatment with only moderate benefits (a reduction of deaths within 14 days by one sixth from $12 \%$ to $10 \%$, which corresponds to avoidance of 20 deaths per 1000 patients treated) might have a substantial public health impact if it were simple and widely practicable. ${ }^{4}$ However, to confirm or refute such moderate benefits reliably requires a trial with a few tens of thousands of patients. ${ }^{5}$ To recruit such large numbers requires the collaboration of many clinicians, so the trial design must be simple enough that busy clinicians can readily participate. ${ }^{5}$

After a systematic review of the medical literature, aspirin and heparin seemed the most promising treatments which could be used widely in acute stroke within the next few years. ${ }^{4}$ The aims of the International Stroke Trial (IST) pilot study were, therefore, to develop the design of a large simple trial that could evaluate the efficacy and safety of aspirin and heparin in patients with acute ischaemic stroke, to examine the validity and feasibility of the design, and to detect or exclude any substantial hazard associated with the trial treatments.

\section{Methods}

ELIGIBILITY

Patients were eligible if symptoms of acute stroke had started within the previous 48 hours. At the start of the pilot phase the exclusion criteria were known intracranial haemorrhage; clear indications for, or clear contraindications to, aspirin or heparin; already dependent in activities of daily living before the stroke; already on aspirin (this criterion was abandoned during the course of the pilot study: see later); already on long term oral anticoagulants; unresponsive coma (this criterion was also abandoned: see later). Computerised tomography, to exclude intracerebral haemorrhage before randomisation, was strongly recommended, but was not compulsory; patients not scanned before randomisation were to be scanned after randomisation where possible.

\section{TREATMENT REGIMENS}

To investigate the effects of aspirin and heparin alone, and in combination, a $3 \times 2$ factorial design was used (table 1). Clinicians were strongly encouraged to adhere to the 
Table 1 Treatment allocations (factorial design)

\begin{tabular}{lll}
\hline (A) $25 \%$ Allocated & (B) $12 \cdot 5 \%$ Allocated & (C) $12 \cdot 5 \%$ Allocated \\
aspirin $\left(300 \mathrm{mg}^{\star}\right)$ & aspirin $\left(300 \mathrm{mg}^{\star}\right)$ & aspirin $\left(300 \mathrm{mg}^{\star}\right)$ \\
avoid heparin & 5000 units subcutaneous & 12500 units subcutaneous \\
heparin twice dailyt & heparin twice dailyt \\
(D) $25 \%$ Allocated & (E) $12.5 \%$ Allocated & (F) $12.5 \%$ Allocated \\
avoid aspirin & avoid aspirin & avoid aspirin \\
avoid heparin & 5000 units subcutaneous & 12500 units subcutaneous \\
& heparin twice dailyt & heparin twice dailyt
\end{tabular}

*Aspirin given by mouth if the patient was able to swallow. If not, it was given either by suppository or intravenously as $100 \mathrm{mg}$ of the lysine salt.

tCalcium heparin was used whenever possible. If not available, sodium heparin was acceptable.

treatment allocation for 14 days or until hospital discharge (if sooner) whenever possible. However, if, in the clinician's opinion, the patient developed either a clear indication for, or a clear contraindication to, the trial treatments, then the patient's treatment could be changed as clinically appropriate. Any nontrial treatments were to be given at the discretion of the responsible clinician and were to be recorded on the discharge form. After the 14 day treatment period, clinicians were encouraged to start aspirin as long term secondary prevention unless contraindicated.

\section{RANDOMISATION AND BASELINE DATA \\ COLLECTION}

Patients were randomised by telephoning the 24 hour randomisation service at the Clinical Trial Service Unit (CTSU), Oxford, UK. During the call, baseline data were requested, which were immediately entered into a computer. Eight questions about the neurological deficit present at the time of randomisation were used to assign the patient's clinical syndrome to one of the four Oxfordshire Community Stroke Project (OCSP) categories ${ }^{67}$ : total anterior circulation syndrome (TACS), partial anterior circulation syndrome (PACS), posterior circulation syndrome (POCS), and lacunar syndromes (LACS). When all the clinical data had been recorded, the randomisation computer (using a minimisation algorithm ${ }^{8}$ to balance the treatment groups with respect to the major prognostic factors of age, conscious level, and OCSP subtype of stroke) generated a treatment allocation. The telephone operator then informed the clinician of that patient's allocated treatment.

FOURTEEN DAY HOSPITAL DATA FORM

At the end of the planned 14 day treatment period (or at death or at discharge from hospital if either occurred sooner), a single paged A4 form was completed by the responsible clinician and sent to the coordinating office in Edinburgh.

SIX MONTH FOLLOW UP

The method used to collect follow up data varied slightly for different countries. In the United Kingdom, Italy, the United States, Portugal, and Australia, six month follow up data were collected by the national coordinator. In these countries, the patient's general practitioner (or responsible hospital clinician) was first contacted by telephone to ensure that the patient was still alive and living at the address recorded on the discharge form. If the patient was not known to be dead, information on functional state, recovery from stroke, and place of residence (for example, in own home, in hospital, or living with relatives) was sought from the patient by postal questionnaire (United Kingdom, Australia), or by telephone interview (Italy, United States, Portugal). The response to the question: "In the last two weeks did you require help from another person for everyday activities?" was used to assess dependency, and to the question "Do you feel that you have made a complete recovery from your stroke?" to assess recovery. These questions, given by postal questionnaire or by telephone call, have been shown to be valid and reliable measures of outcome when compared with a formal assessment by a trained nurse giving the Barthel index and Oxford handicap scale. ${ }^{9}$ Patients in Eire and Finland were assessed by the local hospital coordinator at the hospital outpatients clinic. If the patient had died, the date and likely cause of death were obtained from the patient's general practitioner or hospital doctor.

\section{DATA PROCESSING, ANALYSIS, AND DATA} MONITORING COMMITTEE

Baseline data were electronically transferred from the randomisation computer at Oxford to the main trial data base (dBase IV, Borland International) in Edinburgh. Follow up data were entered twice ("double punching"), with any differences between the two data sets resolved to eliminate data entry errors. Further extensive checks for the internal consistency of the data were also undertaken. Analyses were performed using dBase IV and SPSSPC, ${ }^{10}$ with odds ratios calculated using the Confidence Interval Analysis computer programme. ${ }^{11}$ The prespecified principal analyses for efficacy were the effects of treatment on death within 14 days from any cause, and on "poor outcome" (death or dependency) at six months after randomisation. Safety was assessed by the effect of treatment on fatal and non-fatal intracranial haemorrhage, and on fatal and non-fatal severe extracranial haemorrhages (defined as any haemorrhage severe enough to require blood transfusion or to cause death). The pilot phase of IST was designed only to detect substantial differences in these outcome measures (such as a halving in mortality from about $10 \%$ to $5 \%$ and an increase in intracerebral haemorrhage from about $1 \%$ to $4 \%$ ). The accumulating data were periodically reviewed in strict confidence by an independent data monitoring committee (see acknowledgements). In June 1992, that committee reviewed the data and recommended that, as the balance of benefit and risk remained unclear, the main phase of the IST should proceed with no major change in the trial design. The pilot phase of the trial was closed at 0859 hours on 1 March 1993, and the main phase of the IST started at 0900 hours the same day. As the treatment regimens and allocation remained unchanged in the main phase of the IST, the pilot study results by treatment allocation are to remain confidential and will be combined with the results of the main phase of the study. 
Table 2 Outcome at 14 days and six months in pre-specified subgroups

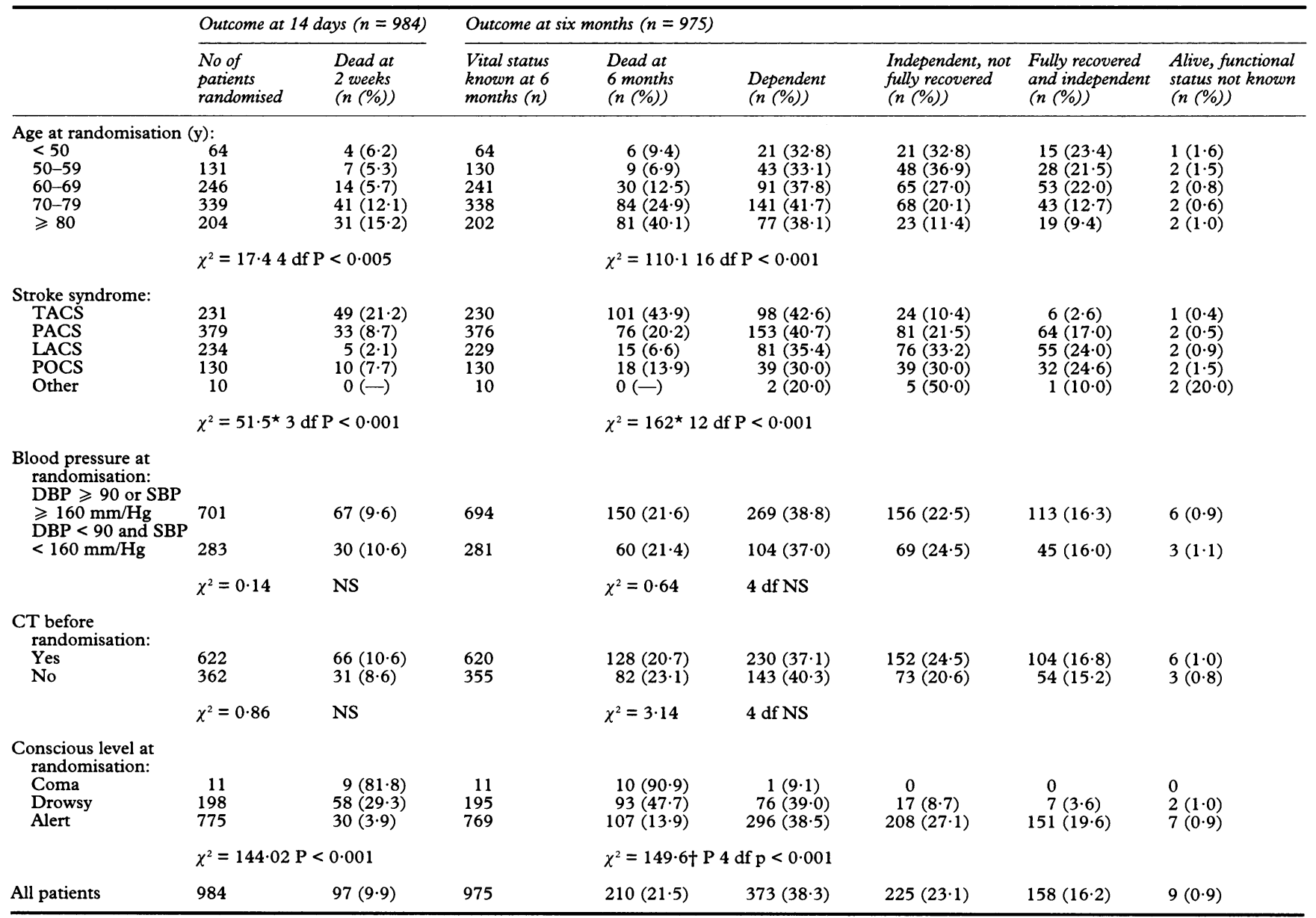

*“Other", group combined with LACS for $\chi^{2}$ test.

$t$ “Coma" and "drowsy" categories combined compared with "alert" for $\chi^{2}$ test.

TACS = Total anterior circulation syndrome; PACS = partial anterior circulation syndrome; POCS = posterior circulation syndrome; LACS = lacunar syndrome

\section{Results}

The pilot study began in Edinburgh on 14 January 1991 and by the close of the study on 1 March 1993, a total of 34 hospitals in nine countries were participating. Nine hundred and eighty four patients were recruited. Centres recruited at an average rate of 2.3 (range $0 \cdot 2-4 \cdot 4$ ) patients per centre per month.

\section{BASELINE CHARACTERISTICS}

There were no significant differences in baseline characteristics between the six treatment groups (data not shown but available on request). Table 2 shows the characteristics of patients at the time of randomisation and their outcome. The average age was 69.9 (SD 11.7, median 71) years, with a range from 23 to 99 : $789(80 \%)$ were aged 60 or over; $543(55 \%)$ were 70 or older; $204(21 \%)$ were 80 or older; and eight $(1.0 \%)$ were over 90 . The time from onset of stroke symptoms (or from waking for strokes with onset during overnight sleep) to randomisation was: 22 patients $(2 \%)$ were randomised within three hours; $117(12 \%)$ within six hours; 325 (33\%) within 12 hours; and 634 (64\%) within 24 hours of symptom onset.

The mean systolic blood pressure was 159 (median 160) $\mathrm{mm} \mathrm{Hg}$ with a range of 90-240 $\mathrm{mm} \mathrm{Hg}$ and the mean diastolic blood pressure was 90 (median 90) $\mathrm{mm} \mathrm{Hg}$ with a range of $30-150 \mathrm{~mm} \mathrm{Hg}$.
PRINCIPAL MEASURES OF EFFICACY (DEATHS WITHIN 14 DAYS AND PROPORTION OF PATIENTS WITH A POOR OUTCOME)

Fourteen day hospital data forms were received for all 984 patients randomised. Ninety seven patients $(10 \%)$ died within 14 days of randomisation. Six month follow up data were available for 975 patients (99\%). Of these 975, nine patients were known to be alive, but their functional state was not known (we continue to seek this information): of the remainder, $210(22 \%)$ patients had died, $373(38 \%)$ were dependent on others for everyday activities, 225 (23\%) were independent but had not fully recovered, and 158 (16\%) were both independent and considered that they had made a complete recovery from their stroke. Thus by six months, 583 patients $(60 \%)$ had a bad outcome after their stroke (were dead or dependent). Table 2 shows the results subdivided by age, subtype of stroke, whether or not CT scanning was performed before randomisation, blood pressure at randomisation, and conscious level.

OTHER MEASURES OF EFFICACY AND SAFETY

(FATAL OR NON-FATAL EVENTS WITHIN 14 DAYS)

Table 3 lists the fatal and non-fatal events explicitly sought, on the 14 day hospital data 
Table 3 Fatal and non-fatal vascular events within 14 days of randomisation *

\begin{tabular}{|c|c|}
\hline \multicolumn{2}{|l|}{ Causes of death within 14 days (n (\%)) } \\
\hline Initial stroke & $68(6 \cdot 9)$ \\
\hline Fatal recurrent stroke: ischaemic (or unknown type) & $4(0 \cdot 4)$ \\
\hline Fatal recurrent stroke: intracranial haemorrhaget & $5(0 \cdot 5)$ \\
\hline Fatal pulmonary embolism & $4(0.4)$ \\
\hline Fatal coronary heart disease & $7(0 \cdot 7)$ \\
\hline Other vascular death & $4(0 \cdot 4)$ \\
\hline Non-vascular death & $5(0 \cdot 5)$ \\
\hline Non-fatal events within 14 days $(n(\%)) \ddagger$ & \\
\hline Recurrent stroke: ischaemic (or unknown type) & $26(2 \cdot 6)$ \\
\hline Recurrent stroke: confirmed intracranial haemorrhaget & $4(0 \cdot 4)$ \\
\hline Non-fatal myocardial infarction & $5(0.5)$ \\
\hline Non-fatal pulmonary embolism & $4(0.4)$ \\
\hline Event clusters $(n(\%))$ & \\
\hline Fatal or non-fatal recurrent stroke: ischaemic (or unknown type) & $30(3 \cdot 0)$ \\
\hline Fatal or non-fatal recurrent stroke: intracranial haemorrhaget & $9(0.9)$ \\
\hline Fatal CHD or non-fatal MI & $12(1 \cdot 2)$ \\
\hline Fatal or non-fatal pulmonary embolism & $8(0 \cdot 8)$ \\
\hline
\end{tabular}

*For patients still in hospital 14 days after randomisation, all fatal and non-fatal events recorded within 14 days are included. For patients discharged from hospital within 14 days, non-fatal events were recorded only for the period in hospital.

tConfirmed by CT or necropsy.

Event occurred within 14 days and patient still alive on day 14.

$\mathrm{CHD}=$ Coronary heart disease; $\mathrm{MI}=$ myocardial infarction

forms. For any patient discharged within 14 days, this analysis included fatal and non-fatal events while in hospital, plus any fatal events occurring between discharge and 14 days. Of the nine patients with a recurrent stroke which was confirmed to be haemorrhagic by CT or necropsy, five $(56 \%)$ died. By contrast, only four deaths occurred within 14 days among the 30 patients $(13.3 \%)$ with a recurrent stroke which was either definitely ischaemic or of unknown type.

OTHER ADVERSE EVENTS AND POSSIBLE SIDE-EFFECTS WITHIN 14 DAYS

Four patients $(0.4 \%)$ had a major extracranial haemorrhage requiring blood transfusion: a subcutaneous haematoma in two patients, a gastrointestinal haemorrhage in one patient, and bleeding from an unspecified site in one. Minor bleeding events were also uncommon with only 35 recorded in 34 patients: haemorrhagic transformation of cerebral infarction (eight); subcutaneous haematoma (six); subcutaneous bruising (four); haematuria (four); haematemesis (two); rectal bleeding (two); bleeding haemorrhoids (one); unspecified gastrointestinal bleeding (one); bladder wall bleeding (one); nose bleed (one); atrial fibrillation (one); extension to initial stroke (one); asymptomatic increase in APPT (one); asthma (one).

\section{OTHER DATA RECORDED ON 14 DAY HOSPITAL} DATA FORM

Compliance with trial treatment

Clinicians were asked to state whether or not patients had adhered to their allocated treatment throughout the intended treatment period (14 days or up to hospital discharge if sooner). Patients who missed only one or two doses were counted as compliant. Based on this definition, good compliance occurred in $419(85 \%)$ of 493 patients allocated aspirin, $213(87 \%)$ of 246 patients allocated low dose heparin, and $189(77 \%)$ of 245 patients allocated medium dose heparin. Only $29(6 \%)$ of $491(6 \%)$ allocated to "avoid aspirin" received some aspirin within the first 14 days and 21 (4\%) of 493 allocated to "avoid heparin" received some heparin within the first 14 days.
Non-trial treatments while in hospital

The most commonly used non-trial treaments were glycerol (168 patients, $17 \%$ ) and calcium channel antagonists (160 patients, $16 \%$ ), with only a few patients receiving haemodilution (21 patients, $2 \%$ ) or steroids (17 patients, $2 \%)$.

\section{Results of $C T$}

At least one scan was performed in 924 (92\%) patients, of which $622(63 \%)$ were performed before randomisation. The results of the first CT were: 310 (34\%) normal or atrophy only; $560(61 \%)$ cerebral infarction; 29 (3\%) primary intracerebral haemorrhage (PICH); 15 (2\%) haemorrhagic transformation of cerebral infarction (HTI); three $(0.3 \%)$ primary or secondary intracerebral tumour; and seven (1\%) other findings. Further information about the rate of haemorrhagic transformation of cerebral infarction with treatment was systematically sought by performing a second scan at 14 days in a special substudy undertaken in two centres (which will be reported separately).

\section{Discussion}

This pilot study confirmed that the study design was practicable in both university hospitals and general hospitals. The size of the pilot study allowed us to make reasonably precise estimates of the event rates in the type of patients to be included in the main study. The observed case fatality of about $10 \%$, suggests that the main study might need to recruit about 20000 patients to detect reliably a $15 \%$ reduction with treatment. ${ }^{2511}$ Given the pilot study rates of fatal and non-fatal vascular events, if 20000 patients were recruited, 2000 would be expected to die within 14 days, about 800 would have recurrent strokes (of which about 150 would be due to cerebral haemorrhage), and 200 would have fatal or non-fatal pulmonary emboli, whereas about 12000 patients would have poor outcome at six months. Such large numbers of events would enable suitably precise estimates of treatment effects to be derived overall and in clinically important subgroups: patients treated within the first few hours compared with those treated later; patients aged under 75 years compared with older patients; patients randomised with or without prior CT; and patients with different subtypes of cerebral infarction.

The pilot study results at two weeks and six months confirmed that the limited data collected in the IST could separate groups at high risk and low risk of a poor outcome. The main predictors of "poor outcome" at six months identified were age, conscious level, and stroke subtype. On the other hand, whether CT was first performed before or after randomisation and the baseline level of blood pressure did not predict "poor outcome" at six months. Detailed prognostic modelling was not appropriate as the number of patients and deaths in the pilot study were small.

The relatively low rate of intracerebral haemorrhage in the pilot study was reassuring 
and helped to exclude a large excess with treatment, but could not reliably exclude a more moderate but perhaps still important excess (10-20 extra per 1000 patients treated). Information on the balance of the risks and benefits of the trial treatments remain confidential while the main study continues, and are available only to the Data Monitoring Committee. The fourth meeting of the Data Monitoring Committee was held on 10 January 1996, at which time 14 day outcome data were available for 15243 patients, and six month data for 12703 patients. The committee reviewed a number of analyses relating to safety and efficacy and recommended continuation of recruitment.

The average recruitment rate in the pilot study was 2.3 patients per hospital per month, with a range of $0 \cdot 1$ to $4 \cdot 4$. Provided the hospitals participating in the pilot study were representative of the sort of hospitals likely to join the main phase of the IST, these results suggest that about 200 hospitals would need to participate for four years in order to recruit 20000 patients. If more hospitals were to collaborate, and if recruitment were at the same rate as in the pilot study, recruitment of 20000 could be completed earlier than 1997, or-perhaps more appropriately-more than 20000 patients could be recruited.

\section{Conclusion}

In the light of these results, there is, as yet, insufficient evidence to support the routine use of aspirin or heparin in patients with acute ischaemic stroke, and recruitment in current trials comparing antithrombotic therapy (using aspirin, heparin, or the combination) with controls should proceed as rapidly as possible.

The international stroke trial pilot study group

Key: COUNTRY (\% of 984 patients randomised by this country): Centre, Hospital, Name of Hospital Medical Coordinator, names of other collaborators (number of patients recruited by this centre).

*Denotes national coordinator.

AUSTRALIA (3\%): Perth, Royal Perth Hospital, Dr G Hankey (29). BELGIUM (4\%): Mons, Clinique St foseph, Dr P Indekeu $^{\star}$ (42). CZECH REPUBLIC (4\%): Hradec Kralove, University Hospital, Dr G Waberzinek (6). Ostrava, City Hospital, Dr C Majvald (17). Plzen, University Hospital, Plzen, Professor Z Ambler ${ }^{\star}$ (12). EIRE: Dublin, St Columcille's Hospital, $\mathrm{Dr} \mathrm{M}$ Crowe $^{\star}$ (5). FINLAND (3\%): Jyvaskyla, Central Hospital, Dr R Fogelholm^ (26). Pori, Pori Hospital, Dr T Jolma (6). ITALY (33\%): Ancona, Ospedale Regionale Torrette Ancona, Professor L Provinciali, Dr S Pelonara, Dr M G Ceravolo (3). Brescia, Ospedali Riuniti Brescia, Dr P Anzola, Dr M Mangoni (1). Como, Ospedale Valduce, Dr M Guidotti (13). Cortona, Ospedale Civile Cortona, Dr R
Migliacci, Dr M Mollaioli, Dr M Aimi, Dr G Conti, Dr C Corbacelli (10). Genova, Universita' di Genova, Professor C Gandolfo, Dr M Conti, Dr R Croce, Dr M Del Sette, Dr C Finocchi (8). Milano, Ospedale Niguarda Ca, Granda, R Sterzi, Dr A Brucato, Dr M De Jiuli, Dr M Rinaldi, Dr G Bottini, Dr G Ferraro (34). Milano, Ospedale Sesto San Giovanni, Dr R Cavestri, Dr M Arreghi, Dr D Gori, Prof E Longhini, Dr R Agosti (8). Modena, Ospedale Civile, Dr G Malferrari, Dr M Bondi, Dr L Melini, Dr W Garuti, Dr F Sani (7). Orvieto, Ospedale Civile $S$. Maria Della Stella, Dr M Franciosini, Dr M Bracaccia, Dr C Gammarota, Dr C Calandri, Dr E Ciorba (5). Pavia, Ospedale di Pavia, Dr G Micieli (4). Perugia, Policlinico Perugia, Dr S Ricci*, Dr M G Celani, Dr E Righetti, Dr P Floridi, Dr G Benemio, Dr E Comparato, Dr E Duca, Dr M Zampolini, Dr T Cantisani, Dr S Biagini, Dr E Vignai, Dr M Guerrieri (107). Sassari, Ospedale di Sassari, Dr A Pirisi (4). Spoleto, Ospedale Civile S Matteo degli Infermi, Dr F Pezzella, Dr N Miele, Dr S Grasselli (10). Todi, Ospedale Civile di Todi, Dr B Biscottini, Dr G Alunni, Dr A Boccali, Dr M Cruciani, Dr M Pacini, Dr R Ibba, Dr R Luccioli (8). Trieste, Ospedale Maggiore, Dr F Chiodo Grandi, Dr L Antoniutti, Dr B Boniccioli, Dr M Gregori, Dr R Valli, Dr N Guerrini, (56). Zingonia, Policlinico San Marco, Dr L Munari, Dr A Perretti, Dr A Mauro (47). PORTUGAL (4\%): Porto, Hospital de St Antonio, Dr M Correia ${ }^{\star}$ (35). UNITED KINGDOM (48\%): Bristol, Frenchay Hospital, Dr C Burns-Cox (32). Edinburgh, Western General Hospital, Dr P Sandercock ${ }^{\star}$, Professor C Warlow, Dr M Dennis, Dr $R$ Lindley, $M$ Proctor, $M$ Mathieson, Mrs F Waddell (134). Glasgow, Western Infirmary and University of Glasgow, Dr K R Lees, E Colquohoun (45). Leeds, St Fames's Hospital, Dr J Bamford (68). Livingstone, St fohn's Hospital, Dr D Farquhar (68). Salford, Hope Hospital, Professor R Tallis, Dr I Scougal, Dr R Meara, (3). Sheffield, Royal Hallamshire Hospital, Dr G Venables, C Holland, H Parry (112). Whipps Cross, Whipps Cross Hospital, Dr K Kafetz (8). UNITED STATES OF AMERICA (1\%): New York, Neurological Institute, Dr R Sacco` Dr D Kargman (11).

\section{Neurosciences Trials Office (Edinburgh)}

Dr P A G Sandercock (clinical coordinator); B Smith (administrative coordinator); Dr R I Lindley (research fellow); Professor C P Warlow (steering committee chairman); J Slattery (statistics); Dr J Wardlaw and Dr R Sellar (neuroradiology); H Fraser (data management); L MacLean, L Robertson, S Honeyman, J MacKenzie, V Scoltock, A Brownlie, G Moody, H Taylor, (clerical and administration); S Anderson, D Charlton, M Cunniffe, I McCrindle (data processing); C McDonald (computer programming). $M$ Livingstone (United Kingdom follow up); F Waddell (nursing). 
Clinical Trial Service Unit, Oxford University

Dr R Collins (Statistics and Neurosciences Trials Office Liaison); Professor R Peto (Statistics); P Dove (Medical Illustration); Dr S Richards (Statistics)

\section{Data Monitoring Committee}

Professor L Wilhelmsen, Goteborg (chairman), Professor J van Gijn, Utrecht, Professor $M$ Harrison, London, and Professor R Peto, Oxford.

\section{Writing Committee}

Dr R Lindley, Dr P Sandercock, Professor C Warlow, Mr J Slattery, Ms B Smith, Dr R Collins.

This pilot study could not have been undertaken without the cooperation of the patients who agreed to participate. Many of the patients were assessed by junior hospital doctors or appropriately trained nurse practitioners; their collaboration (and that of many other people too numerous to list) made this study possible. The staff of the Neurosciences Trials Unit involved in this study are supported by grants from the Medical Research Council and the Stroke Association. Additional grants to support the pilot study were provided Additional grants to support the pilot study were provided Dale Fund, Edinburgh University, and the Kennedy Bequest.
The enteric coated aspirin tablets used by United Kingdom centres were provided by Lilly Industries UK, and the subcutaneous heparin supplies were provided by CP Pharmaceuticals (and subsequently Fisons UK Ltd). The design, conduct, and analysis of this study were undertaken independently of the sponsors of the research.

1 Sandercock $P$, Willems $H$. Medical treatment of acute ischaemic stroke. Lancet 1992;339:537-39.

2 Yusuf S, Collins R, Peto R. Why do we need some large, simple randomised trials? Stat Med 1984;3:409-20.

3 Bamford J, Sandercock P, Dennis M, Burn J, Warlow C. A prospective study of acute cerebrovascular disease in the community: The Oxfordshire Community Stroke Project-1981-6 2. Incidence, case fatality, and overall outcome at one year of cerebral infarction, primary intracerebral haemorrhage and subarachnoid haemorrhage. F Neurol Neurosurg Psychiatry 1990;53:16-22.

4 Sandercock PAG, van den Belt AGM, Lindley RI, Slattery J. Antithrombotic therapy in acute ischaemic stroke: an Neurosurg Psychiatry 1993;56:17-25.

5 ISIS-2 Collaborative Group. Randomised trial of intravenous streptokinase, oral aspirin, both, or neither venous streptokinase, oral aspirin, both, or neither infarction: ISIS-2. Lancet 1988;ii:349-60.

6 Bamford J, Sandercock P, Dennis M, Burn J, Warlow C. Classification and natural history of clinically identifiable subtypes of cerebral infarction. Lancet 1991;337:1521-6.

7 Bamford J. Clinical examination in diagnosis and subclassification of stroke. Lancet 1992;339:400-2.

8 White SJ, Freedman LS. Allocation of patients to treatmen groups in a controlled clinical study. Br F Cancer 1993, 37:849-57.

9 Lindley RI, Waddell F, Livingstone M. Can simple questions assess outcome after stroke? Cerebrovasc Dis 1994;4: 314-24

10 Norusis P. Statistics package for the social sciences (SPSS$P C)$. Chicago: SPSS Inc, 1988.

11 Gardiner $M$, Altman $G$. Statistics with confidence. London: BMJ Publishing Group, 1989. 